\title{
Spectropolarimetry and polarization-dependent fringes ${ }^{\star}$
}

\author{
M. Semel ${ }^{\star \star}$ \\ LESIA, Observatoire de Paris, section de Meudon, 92195 Meudon, France
}

Received 3 January 2002 / Accepted 26 September 2002

\begin{abstract}
An account is given of the formation of polarization-dependent fringes, the "enemies" of spectropolarimetry, present practically in all polarization devices. Typically, multiple reflections give rise to secondary beams coherent with the main beam, but with wavelength-dependent phase differences. Polarized fringes may appear in any particular Stokes parameter or their combinations, and may (or may not) be seen in the intensity. The mechanism of formation of fringes in polarization is demonstrated. Fringes of analyzers, beam-splitter and retarders are evaluated. Flat-fielding techniques are discussed and ways for the removal of polarized fringes are suggested. Techniques that are efficient in removing fringes in intensity may be inadequate or insufficient for polarization. Anti-reflection coating, for instance, may reduce fringes, but not below $10^{-3}$ of the intensity. This level would often be acceptable for intensity measurements but not necessarily for polarization, where one may often need to evaluate signals down to the level of $10^{-5}$, as in the observation of resonance line polarization and the second solar spectrum. Flat-fielding, by observing disk center, may be satisfactory to remove fringes for solar observations but this is not appropriate for stellar observations. The understanding of how these fringes are formed is essential both for the design of polarimeters and for their proper use.
\end{abstract}

Key words. magnetic fields - polarization - instrumentation: polarimeters - techniques: polarimetric

\section{Introduction}

The phenomenon to be treated in this paper has been encountered by almost any observer in the field of high resolution spectropolarimetry, but the theory remained obscure for several reasons, including beliefs. We must clarify some aspects, doubts and erroneous convictions before we may proceed.

\subsection{Prologue: Fringes or channels?}

The topic treated in this paper has not been addressed in the literature except for one contribution in a workshop, by Lites (1991), apparently not known to most of the astronomers in the field of spectropolarimetry. We must admit that even the terminology is a problem. What should we call these periodic spurious signals? Fringes or channels?

These terms, in general, point to particular structures or shapes and were borrowed by opticians from elsewhere (fringes of rug, hair etc.) in order to denominate similar forms in interference experiments. There are no formal definitions for these terms beyond the common use.

Both terms describe various results of light interference which look periodic, but channels appear in the spectrum as

\footnotetext{
* Appendix $\mathrm{C}$ is only available in electronic form at http://www . edpsciences.org

$\star \star$ e-mail: Meir.semel@obspm.fr
}

a function of the wavelength only (as in the case of FabryPerot etalon combined with a spectrograph, for instance) while fringes are attributed to the periodic features function of space, as in Young's experiments; colors are seen as well, but with a weak wavelength dependence. (However, Hecht 1987, p. 379, uses the term fringes also in the case of Fabry-Perot etalon! The term Channels is not mentioned at all in his book.) See Jenkins $\&$ White (1957) for a description of channeled spectrum. The features we are treating in the present paper are functions of both $\lambda$ and space, and could be denominated Frinnels or better Chingpols as they are often periodic in both dimensions and are also polarized. In any case it is clear that these periodic features are neither pure Channels nor pure Fringes. What to call them? We leave this important question to the academy. Leroy (2002) clarified the semantics as follows: Multiple reflections in a plane parallel plate placed at the pupil of the spectrograph would create pure channels, i.e. dependent only on $\lambda$ and thus oriented perpendicular to the dispersion; practically this will be still the case even if the plate were perfect and placed anywhere in the beam. However, if the plate is not perfectly plane parallel and is placed at the image (the slit plane of a spectrograph), the features may be periodic in an oblique direction, i.e. function of both $\lambda$ and the spatial coordinate along the slit. If the plate is a retarder, then the features are polarized as well. In this paper, whenever the term fringes is used we mean fringes-channels features. 


\subsection{Digression: Polarization and Fresnel-Arago rule for the appearance of fringes}

The rule in question can be found in "Optics" p. 339 (Hecht 1987). A coverage of this topic is given by Wood (1911), who mentions five rules, including those concerning occurrence of polarized fringes. The same author (Wood 1904) performed a nice experiment in the same field. Polarized fringes are encountered in the context of "polariscopes à franges" as well (Leroy 1998 , p. 44). The misunderstanding of this historical rule is certainly a very serious flaw to be treated in some detail.

We therefore return to the pure fringes as seen in Young's experiment (Jenkins \& White 1957, Fig. 13F, p. 236). Using Stokes formalism, we will treat in detail the case where the two interfering beams are polarized in two orthogonal states (Sect. 2). Objectively, we are not concerned by this rule. Fresnel and Arago meant only pure fringes, beyond any spectroscopy! The fringes we are interested in are of quite a different nature. However, more than once, the interpretation of the spurious and periodic spectropolarimetric signals in terms of polarization dependent fringes faced serious objections.

\subsection{The astronomical context}

The use of spectropolarimetry in astronomy is related mainly to the measurements of magnetic fields in the sun and stars. Magnetic fields play an important role in solar activity and therefore have been subject to much research since the beginning of the last century. In recent years, stars were used to prove that solar type activity is very important in young stars.

Very particular tools were developed for solar magnetic field measurements, like radiative transfer equations of polarized light starting with the pioneer work of Unno (1956). A sophisticated code for the integration of these equations was given by Rees et al. (1989). Numerous studies of solar Zeeman effect have improved and refined Stokes polarimetry, see for instance Skumanich \& Lites (1987).

However, fringes in spectropolarimetry still pose a serious problem. This paper is a study of their origin and their phenomenology in spectropolarimetry both in stellar and solar observations.

One of the main difficulties in spectropolarimetry is parasitic light. Whatever the source of this parasitic light, if it is coherent with the main beam, fringes will appear in the polarimetric signals and may have important consequences for data analysis.

There are only a few papers that deal with the problem of multiple reflections inside a quarter wave plate. Lites (1991) treats the case of the Advanced Stokes Polarimeter. Holmes (1964) calculated the modifications of the phase retardation due to multiple reflections. Makita et al. (1982) discussed fringes due to multiple reflection in a quarter wave plate. Nevertheless, the problem of polarization-dependent fringes has remained baffling and still requires urgent elucidation.

The precision attained in the intensity spectrum measurements is affected by the presence of fringes but also by other phenomena such as poor calibration, undetermined scattered light, difficulties to determine the true continuum, or spectral blends. On the other hand, very weak polarization signals can often be observed, however, their polarization-dependent fringes may completely swamp these signals. Various efficient techniques for removing fringes in intensity are insufficient for polarization. For solar observations, it is often possible to flatfield out fringes by the employment of unpolarized light at the disk center or the use of de-focused solar images. This method has the disadvantage of being time consuming, which is undesirable in the case of good seeing since flat-fielding should be done close to the time of observation. Thus one needs to consider alternative ways of reducing fringes since flat-fielding through disk center observations, efficient for the sun, is not appropriate for stellar observations. Finally, all flat-fielding with artificial light sources is unsatisfactory beyond levels of $10^{-3}$ of the intensity. It is still very difficult to produce (artificial) terrestrial light for flat-fielding with the quality of the celestial one. Spectropolarimetry must take into consideration the sources of these fringes and the ways of reducing them, by methods other than flat-fielding.

\subsection{The Stokes formalism}

Stokes vectors are obtained through statistical averaging over the coherences of the electric components of the light. The reader may find the definition of the Stokes vector and discussions in numerous papers and it will not be repeated here in detail. We want only to stress here two points concerning this formalism. First, the averaging may depend on the spatial, temporal, spectral or angular resolutions; while a perfectly quasimonochromatic beam may be unpolarized, monochromatic light (an academic situation, indeed) is always completely polarized. Second, uncorrelated beams (no phase relation) may be added easily, presenting an exceptional advantage of this formalism; indeed, in many cases it is well justified and it has been applied abundantly in astrophysics. This applies to the fields of radiative transfer of polarized light, solar magnetographs, devices for spectropolarimetry, calculations of instrumental polarization, etc. (See for instance del Toro Iniesta et al. 2000 for a lucid assessment of modern solar Stokes polarimeters; also see references therein for the design of these instruments.)

Thus, most of the entities in spectropolarimetry are well represented in the Stokes regime. To many it seems that everything is covered by this formalism. However, not all the physics of light is present in this space! There are some phenomena, even very simple ones, like fringes, that have not received the necessary attention!

Indeed, one of the difficulties encountered in spectropolarimetry is the appearance of various kinds of fringes in any combination of the Stokes parameters. For fringes in a nighttime telescope see for instance Donati et al. (1999). Themis, (Mein et al. 1985), a recent French-Italian solar telescope, encountered this problem as well. In general and erroneously enough, fringes are considered as a technical problem. Indeed they present a puzzle in the field of spectropolarimetry, eventually assumed to be due to a defect of manufacture, and are believed to be removable by some technical devices. We consider 
that the correct strategy would be to take them into account in the initial design.

\subsubsection{Beyond the Stokes regime}

In optics we can distinguish three aspects: 1) the wave propagation of light where phase and amplitude are important; 2) incoherent addition of light where phase relation is ignored - the realm of Stokes vectors and Mueller matrices; and 3) the measurements of light and Stokes polarimetry. Most work on solar and stellar polarimetry focuses on the second one where fringes are neglected. Retarders and beamsplitters, in common use in polarimetry, are therefore succintly described by Mueller matrices. But optical companies do not sell soft matrices, they sell hard optics, whose behavior is only approximated by the common matrices. We need an excursion out of the Stokes formalism to calculate fringes and fortunately, as will be shown, we may go back and integrate them into the Mueller matrices. This may open the way to deal correctly with polarimeters and spectropolarimetry free of fringes.

Fringes and light interference are not the only exception. The Polarimetry and the measurements of light, is part of the third aspect which is beyond the scope of the present paper.

\subsection{The structure of the paper}

As a preliminary step we must mention the Fresnel-Arago rule (Sect. 2) to make sure that this important condition for the appearance of fringes has no bearing on our "fringes", which are of quite different origin (see Sects. 3 and 4).

The following Sect. 3 treats scalar fringes and shows how very a faint beam may produce serious spurious signals in the spectrum. This problem is not encountered in broad-band spectroscopy or polarimetry. The origin of the (parasitic) beam is not specified, it can be due to multiple reflections in a plane parallel plate or to scattered light from the edges of the optical piece or even to defects in the optics. Essentially, in an isotropic medium, all states of polarization may propagate without any change except for light losses, oblique reflection excluded. No polarization effects are expected here. However, the significant effects of a faint parasitic light are valid also for the polarization signals as well.

Section 4 introduces the polarized fringes, eventually produced in uniaxial crystals, polarization analyzers or retarders. Contrary to the experiments described when discussing the Fresnel-Arago condition, such fringes are produced independently in each one of the eigenstates of the light in the optical elements. No phase relation between these eigenstates is necessary for the formation of fringes.

The term eigenstate of the light needs to be explained. Also we need to show how the formation of scalar fringes can help us to understand the formation of non-scalar fringes in an anisotropic medium for which we will need to apply the rules of wave propagation of polarized light. The transition from scalar to polarized fringes is justified in Sect. 4.1.

Typically, we concentrate on uniaxial crystals, an anisotropic medium, where the ordinary and extraordinary rays have different laws of propagation. There are, therefore, two eigenstates of polarized light, ordinary and extraordinary. They propagate independently of each other and create their own system of fringes. The two states are orthogonal linear polarizations of the light. The sum of the respective fringes will appear in the intensity spectrum, and the difference in the polarization spectrum. Since the frequencies, the phases or the amplitudes of the two fringe systems are different, polarized fringes may be created. No interference between the two different states of polarization is considered here. Mueller matrices including the parasites effects will be derived.

Commercial achromatic retarders are composed of two different uniaxial crystals and deserve a Sect. 5 devoted to this polarimetric tool in very common use. A knowledge of the characteristics of the commercial retarders may help us to propose procedures to remove spurious polarimetric signals. This topic is beyond the scope of the present paper and will be the subject of a forthcoming one.

\section{Fringes in interference of polarized beam and the Fresnel-Arago rule}

\subsection{The fundamental rule for interference with two beams experiments and Young's setup}

The two beams should have a phase relation for fringes to show up.

Practically the only way to satisfy this condition is to split a light beam and make the split beams interfere. There are many ways to split the beams. Young used a source $S$ that illuminated two adjacent slits, S1 and S2. The resultant diverging beams have therefore a phase relation and interfere. Fringes appear on the screen (Fig. 1). For the same purpose, Fresnel invented the double mirrors and bi-prism techniques. Lloyd performed a similar situation with his mirror. Assuming that the reader is familiar with these experiments, we turn to the case of polarized beams. In Fig. 1, the polarizers p1, p2 are now put in front of the slits S1, S2 respectively. The most interesting case is certainly when the polarizers are set to orthogonal states.

Now, all depends on the nature of the light emitted by the source $\mathrm{S}$. If it is natural, no fringes may appear, as in this case the orthogonal light vibrations are completely independent. No phase relation occur in such a case.

If the light beam at $\mathrm{S}$ is polarized, there is a phase relation between the orthogonal states.

We assume that $\mathrm{p} 1$ is a linear polarizer oriented parallel to the plane of the figure and yielding $+Q, \mathrm{p} 2$ is orthogonal to $\mathrm{p} 1$ and therefore perpendicular to the plane of the figure and yielding $-Q$. If the light at $\mathrm{S}$ were circularly polarized, a phase relation should exist between the two vibrations at $\mathrm{p} 1$ and $\mathrm{p} 2$, i.e. a relative delay of $90^{\circ}$.

To calculate the general case of partially polarized elliptical light, we must turn to quasi-monochromatic light. We therefore assume that the light has some spectral extension and therefore contains a range of wavelengths $\lambda_{k}$ with the corresponding amplitudes $A_{x, k}$ and $A_{y, k}$ for the polarization orientations $x$ and $y$ respectively. The phases are given naturally by $\varepsilon_{x, k}$ and $\varepsilon_{y, k}$ respectively. The two corresponding linear components 


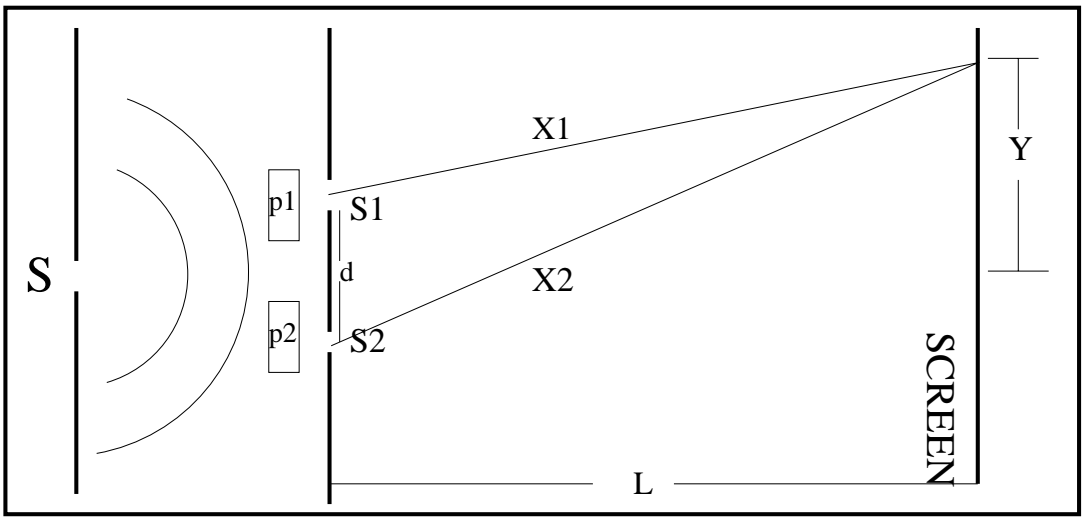

Fig. 1. A sketch of Young's setup for Fringes: (dimensions are out of proportions) $\mathrm{S}$ is a source of light projected onto the slits S1 and S2, that are, therefore, coherent sources. But the polarizers P1 and $\mathrm{P} 2$ in front of the slits select perpendicular components of the electric vector. This modifies completely the possible interferences on the screen. See text for calculations. are therefore given by a sum of plane-waves propagating in the $z$ direction:

$$
\begin{aligned}
& E_{x}=\sum_{k} A_{x, k} \exp \left[i\left(2 \pi(c t-z) / \lambda_{k}-\varepsilon_{x, k}\right]\right. \\
& E_{y}=\sum_{k} A_{y, k} \exp \left[i\left(2 \pi(c t-z) / \lambda_{k}-\varepsilon_{y, k}\right]\right.
\end{aligned}
$$

$$
\begin{aligned}
X 2 & =X 1+\Delta X \\
\varepsilon_{k}^{(2)} & =\varepsilon_{k}^{(1)}+2 \pi \Delta X / \lambda_{k}=\varepsilon_{k}^{(1)}+\Delta \varepsilon_{k} .
\end{aligned}
$$

(2) We now turn to the interference of the two beam experiment with $\mathrm{p} 1$ and $\mathrm{p} 2$ corresponding to the $x$ and $y$-polarizations, and use the parameters in Fig. 1. At point $\mathcal{Y}$ on the screen, the beams have extra paths $X 1, X 2$. We use Eqs. (1) and (2), assuming the same weakening, $W$, for all amplitudes. For simplicity, we keep the same $z$ in both equations and we get

$$
\begin{aligned}
I & =<E_{x}^{*} E_{x}>+<E_{y}^{*} E_{y}> \\
Q & =<E_{x}^{*} E_{x}>-<E_{y}^{*} E_{y}> \\
U & =<E_{x}^{*} E_{y}>+<E_{x} E_{y}^{*}> \\
V & =i^{-1}\left(<E_{x}^{*} E_{y}>-<E_{x} E_{y}^{*}>\right)
\end{aligned}
$$

were $<>$ means time averaging and $E_{x}^{*}$ means the complex conjugate of $E_{x}$. The product $E_{x}^{*} E_{x}$ contains terms like $\cos \left(2 \pi c t\left(\lambda_{k}^{-1}-\lambda_{j}^{-1}\right)\right)$. When $j \neq k$ these terms are (time)averaged to zero. We can finally write:

$$
\begin{aligned}
& I=\sum_{k} A_{x, k}^{2}+\sum_{k} A_{y, k}^{2} \\
& Q=\sum_{k} A_{x, k}^{2}-\sum_{k} A_{y, k}^{2} \\
& U=2 \sum_{k} A_{x, k} A_{y, k} \cos \left(\varepsilon_{x, k}-\varepsilon_{y, k}\right) \\
& V=2 \sum_{k} A_{x, k} A_{y, k} \sin \left(\varepsilon_{x, k}-\varepsilon_{y, k}\right) .
\end{aligned}
$$

From the geometry of Fig. 1 one may calculate the phases as modified along the paths $X 1$ and $X 2$ :

$$
\begin{aligned}
& {[X 1]=\left(L^{2}+(Y-d / 2)^{2}\right)^{1 / 2}} \\
& {[X 2]=\left(L^{2}+(Y+d / 2)^{2}\right)^{1 / 2} .}
\end{aligned}
$$

We then get the corresponding phase changes:

$\varepsilon_{k}^{(1)}=2 \pi[X 1] / \lambda_{k}$

$\varepsilon_{k}^{(2)}=2 \pi[X 2] / \lambda_{k}$.

$$
\begin{aligned}
& E_{x}=W \sum_{k} A_{x, k} \exp i\left[2 \pi(c t-z) / \lambda_{k}-\varepsilon_{x, k}\right] \\
& E_{y}=W \sum_{k} A_{y, k} \exp i\left[2 \pi(c t-z) / \lambda_{k}-\varepsilon_{y, k}\right] .
\end{aligned}
$$

We replace $\left(2 \pi c / \lambda_{k}\right)$ by the angular frequency $\omega_{k}$ and we further simplify the last equations by substituting $\omega_{k} z$ by the retardation effects of $\varepsilon_{k}^{(1)}$ and $\varepsilon_{k}^{(2)}$ and get for the screen point $\mathcal{Y}$ :

$E_{x}(\mathcal{Y})=W \sum_{k} A_{x, k} \exp i\left(\omega_{k} t-\varepsilon_{x, k}-\varepsilon_{k}^{(1)}\right)$
$E_{y}(\mathcal{Y})=W \sum_{k} A_{y, k} \exp i\left(\omega_{k} t-\varepsilon_{y, k}-\varepsilon_{k}^{(2)}\right)$.

We now reconstruct the Stokes vector at the point $\mathcal{Y}$ on the screen.

$$
\begin{aligned}
I_{\mathrm{S}}(\boldsymbol{y}) & =W^{2} I \\
Q_{\mathrm{S}}(\boldsymbol{y}) & =W^{2} Q \\
U_{\mathrm{S}}(\boldsymbol{y}) & =W^{2} 2 \sum_{k} A_{x, k} A_{y, k} \cos \left(\varepsilon_{x, k}-\varepsilon_{y, k}-\Delta \varepsilon_{k}\right) \\
V_{\mathrm{S}}(\boldsymbol{y}) & =W^{2} 2 \sum_{k} A_{x, k} A_{y, k} \sin \left(\varepsilon_{x, k}-\varepsilon_{y, k}-\Delta \varepsilon_{k}\right) .
\end{aligned}
$$

Since we considered a quasi-monochromatic light, i.e.

$\left(\lambda_{\max }-\lambda_{\min }\right) /\left(\lambda_{\max }+\lambda_{\min }\right) \ll 1$,

we may define an average of $\Delta \varepsilon_{k}$ by using an average of $\lambda_{k}$, i.e. $\bar{\lambda}: \Delta \varepsilon=2 \pi \Delta X / \bar{\lambda}$.

We rewrite the trigonometric terms in Eqs. (24) and (25):

$$
\cos \left(\varepsilon_{x, k}-\varepsilon_{y, k}\right) \cos (\Delta \varepsilon)+\sin \left(\varepsilon_{x, k}-\varepsilon_{y, k}\right) \sin (\Delta \varepsilon)
$$$$
\sin \left(\varepsilon_{x, k}-\varepsilon_{y, k}\right) \cos (\Delta \varepsilon)-\cos \left(\varepsilon_{x, k}-\varepsilon_{y, k}\right) \sin (\Delta \varepsilon)
$$ 




Fig. 2. Modifications of the environments around the slits S1 and S2: in $\mathbf{2 A}$ (on the left) we add a polarizer $\mathrm{P} 3$ in front of $\mathrm{P} 1$ and $\mathrm{P} 2$ oriented at $45^{\circ}$ with them and a similar one behind the slits S1 and S2. Should that guarantees the appearance of fringes on the screen? In $\mathbf{2 B}$ (on the right) we modify Fig. 1 by removing the polarizers P1 and P2 and replace them by two linear retarders $\pm \lambda / 4$ in perpendicular (or orthogonal) orientations. How would that affect the appearance of Fringes?

and we obtain:

$$
\begin{aligned}
I_{\mathrm{S}}(\mathcal{Y}) & =W^{2} I \\
Q_{\mathrm{S}}(\mathcal{Y}) & =W^{2} Q \\
U_{\mathrm{S}}(\mathcal{Y}) & =W^{2}(U \cos (\Delta \varepsilon)+V \sin (\Delta \varepsilon)) \\
V_{\mathrm{S}}(\mathcal{Y}) & =W^{2}(V \cos (\Delta \varepsilon)-U \sin (\Delta \varepsilon))
\end{aligned}
$$

The setup discussed is equivalent to the effect of a linear retarder, with the retardation $\Delta \varepsilon$, well represented by the following Mueller matrix:

$\Theta(\boldsymbol{y})=W^{2}\left(\begin{array}{cccc}1 & 0 & 0 & 0 \\ 0 & 1 & 0 & 0 \\ 0 & 0 & \cos (\Delta \varepsilon) & \sin (\Delta \varepsilon) \\ 0 & 0 & -\sin (\Delta \varepsilon) & \cos (\Delta \varepsilon)\end{array}\right)$.

When $\Theta(\mathcal{Y})$ operates on the Stokes vector $[I, Q, U, V]$, it produces the same result as in Eqs. (28)-(31). Obviously $I_{\mathrm{S}}$ and $Q_{\mathrm{S}}$ are fringe-free in agreement with the rule in question. However fringes appear in $U_{\mathrm{S}}$ and $V_{\mathrm{S}}$. They can be seen both by using adequate polarimetric tools and they are not in the same phase on the screen. However, the quantity $\left(U_{\mathrm{S}}^{2}+V_{\mathrm{S}}^{2}\right)$ is fringe-free.

In Fig. 2A, for example, the addition of the 2 polarizers $\mathrm{P} 3$ allow us to recover the polarized fringes in intensity.

\subsection{Young's setup with retarders}

We consider now Fig. 2B. The orientation of the slow axis of the $\lambda / 4$ plate at $\mathrm{S} 1$ is made to coincide with the $x$-orientation and with the orientation of the fast axis of the $\lambda / 4$ plate at $S 2$. (the two identical $\lambda / 4$ are at orthogonal orientations). The $y$ orientation is therefore the fast, slow axis of the two plates at $\mathrm{S} 1$, $\mathrm{S} 2$ respectively.

The quasi-monochromatic light is now assumed unpolarized, i.e. no coherence between $E_{x}$ and $E_{y}$ but $E_{x 1}$ and $E_{x 2}$ are still coherent. Equation (1) now reads:

$E_{x 1}=\sum_{k} A_{x, k} \exp i\left(2 \pi(c t-z) / \lambda_{k}-\varepsilon_{x, k}-\phi_{1, x}\right)$

$$
E_{x 2}=\sum_{k} A_{x, k} \exp i\left(2 \pi(c t-z) / \lambda_{k}-\varepsilon_{x, k}-\phi_{2, x}\right) .
$$

All the terms are those as before, plus $\phi_{1, x}$ which stands for the $x$ retardance due to the $\lambda / 4$ plate at the slit $\mathrm{S} 1$, and $\phi_{2, x}$ which stands for the $x$ retardance due to the $\lambda / 4$ plate at the slit $\mathrm{S} 2$. When the beams reach the screen at $\mathcal{Y}$, we have to incorporate $\varepsilon_{k}^{(1)}$ and $\varepsilon_{k}^{(2)}$ into the expressions of $E_{x 1}$ and $E_{x 2}$ respectively. With the same conventions as in Eq. (20), we write:

$E_{x 1}(\mathcal{Y})=W \sum_{k} A_{x, k} \exp i\left(\omega_{k} t-\varepsilon_{x, k}-\phi_{1, x}-\varepsilon_{k}^{(1)}\right)$
$E_{x 2}(\boldsymbol{y})=W \sum_{k} A_{x, k} \exp i\left(\omega_{k} t-\varepsilon_{x, k}-\phi_{2, x}-\varepsilon_{k}^{(2)}\right)$
$E_{x}(\boldsymbol{y})=E_{x 1}(\mathcal{Y})+E_{x 2}(\mathcal{Y})$

The intensity at the screen due to the $x$ vibrations, i.e. $I_{x}=$ $<E_{x}(\mathcal{Y}) E_{x}^{*}(\mathcal{Y})>$, is:

$$
\begin{aligned}
\frac{I_{x}}{2 W^{2}}= & \sum_{k}\left(A_{x, k}\right)^{2}\left[1+\cos \left(\phi_{1, x}-\phi_{2, x}+\varepsilon_{k}^{(1)}-\varepsilon_{k}^{(2)}\right)\right] \\
& +<\sum_{k \neq j} A_{x, k}\left[A_{x, j} \exp i\left(\omega_{k}-\omega_{j}\right) t+\cdots\right]>
\end{aligned}
$$

The last term is the time average of a sin and cos functions of the time and thus averages out. As before we approximate $\varepsilon_{k}^{(2)}-$ $\varepsilon_{k}^{(1)} \approx-\Delta \varepsilon$ Also, in virtue of our assumption of identical and orthogonal $\lambda / 4$ plates, we have the following phase relations:

$\phi_{1, x}-\phi_{2, x}=\pi / 2$

$I_{x}=\left(I_{x, \max } / 2\right)(1+\cos (\Delta \varepsilon+\pi / 2))$.

Note that, $E_{y 1}$ and $E_{y 2}$ are coherent as well and therefore

$\phi_{1, y}-\phi_{2, y}=-\pi / 2$.

Thus, the intensity of the $y$ component is given by:

$I_{y}=\left(I_{y, \max } / 2\right)(1+\cos (\Delta \varepsilon-\pi / 2))$.

In the absence of polarization in the incoming beam:

$I_{x, \max }=I_{y, \max }=I_{\max }$.

The Stokes vector is given by:

$I(\mathcal{Y})=I_{x}+I_{y}=I_{\max }$

with no fringes in the intensity. However,

$Q(\mathcal{Y})=I_{x}-I_{y}=I_{\max } \cos (\Delta \varepsilon+\pi / 2)$

which means that polarized fringes in the linear polarization appear in $Q$. It is easy to check that

$U(\mathcal{Y})=V(\mathcal{Y})=0$.

Fringes appear therefore in the linear polarization parallel to the axis of the $\lambda / 4$ plates.

Let us repeat the previous experiment with linear retarders, of retardance $\pm \theta$ and with the same orientation as before. Equations (40) and (42) are replaced by:

$$
\begin{gathered}
I_{x}=I_{y, \max }(1+\cos (\Delta \varepsilon+\theta)) / 2 \\
I_{y}=I_{y, \max }(1+\cos (\Delta \varepsilon-\theta)) / 2 .
\end{gathered}
$$


And Eqs. (44) and (45) by

$$
\begin{aligned}
& I(\mathcal{Y})=I_{x}+I_{y}=I_{y, \max }(1+\cos (\Delta \varepsilon) \cos \theta) \\
& Q(\mathcal{Y})=I_{x}-I_{y}=-I_{y, \max } \sin (\Delta \varepsilon) \sin \theta
\end{aligned}
$$

Note that the fringes in $Q(\mathcal{Y})$ are shifted relatively to those in $I(\mathcal{Y})$ by $90^{\circ}$. They shift from one to the other of the Stokes parameters according to the value of $\theta$. However the total intensity of the fringes is invariant.

$\left[\overline{\left(Q(\mathcal{Y})^{2}+\left(I(\mathcal{Y})-I_{y, \max }\right)^{2}\right.}\right]^{1 / 2}=\frac{I_{y, \max }}{\sqrt{2}}$.

The fringes may switch between $I, Q, U$ and $V$, sometimes as a function of wavelength. This phenomenon is the principal topic of the present paper.

\section{Scalar fringes}

In this section we provide a simple demonstration of how the fringes in question appear. We consider the case in which the speed of light at a given wavelength is independent of the type of vibrations of the electric vector (circular, linear, or elliptical) This also implies the same index of refraction for all the states of polarization. In such a case, the fringes are the same for all the components of the Stokes vector and will be referred to as "scalar fringes".

Under certain circumstances (to be treated in the next section), the speed of light, and therefore the refraction indices, may be different for different types of vibrations of the electric vector. This is the case of double refractive media, where polarization dependent fringes may appear.

Let us examine the interference of two light beams: a principal one and a secondary one. It is shown that even a weak and apparently insignificant secondary beam may lead to quite significant spurious signals. At a given point, the electric fields in the principal and secondary beams are denoted by $A \cos (\omega t)$ and $B \cos (\omega t-\phi)$ respectively where the usual symbols for amplitudes, angular frequency and time are used. The phase retardance of the secondary beam is denoted by $\phi$.

The addition of the electric vibrations of the two beams yields

$E=A \cos (\omega t)+B \cos (\omega t-\phi)$.

The total intensity is given, as usually, by

$$
\begin{aligned}
I & =<E^{2}>=A^{2} / 2+B^{2} / 2+A B \cos \phi \\
& =I_{A}+I_{B}+A B \cos \phi .
\end{aligned}
$$

As an illustration, consider the case where the secondary beam is $10^{6}$ times weaker than the principal one $\left(I_{B}=10^{-6} I_{A}\right)$ and therefore may be looked as negligible and bellow the treshold of detection. However, $B=10^{-3} A$ and therefore the third term $A B \cos \phi$ has an amplitude of $2 \times 10^{-3} I$ and cannot be neglected.

The phase delay $\phi$ of the secondary beam may be wavelength dependent, as will be shown in the next subsection, thus explaining the appearance of fringes in wavelength or channels.

\section{Amplitude and phase calculations}

For the evaluation of fringes in polarization, later on we will need to calculate changes in amplitude and phase caused by the secondary beam. We rewrite Eq. (52) in complex notation

$E=A \exp (i \omega t)+B \exp (i \omega t-\phi)$.

Consider now the expression:

$E=C \exp [i(\omega t-\psi)]$.

Equating these last two equations we have

$A+B \exp (-i \phi)=C \exp (-i \psi)$

which allow us to evaluate $C$ and $\psi$. By equating the imaginary parts,

$B \sin \phi=C \sin \psi$

and using the relation $|B / C| \ll 1$ (as in last subsection example), leading to $|\sin \psi| \ll 1$, one finds the phase modification

$\psi \approx \sin \psi=\frac{B}{C} \sin \phi$.

To complete this exercise we calculate the amplitude $C$ by equating also the real part,

$A+B \cos \phi=C \cos \psi$

to obtain

$C^{2}=A^{2}+B^{2}+2 A B \cos \phi$

corresponding to Eq. (53) We note the change in the amplitude of the electric oscillation:

$\frac{C}{A} \approx 1+\frac{B}{A} \cos \phi$.

Equations (58) and (61) that give the changes in phase and amplitude are the basic tools to evaluate fringes of all kinds.

\subsection{Fringes in a plane parallel plate}

Consider a beam of light passing through a plane parallel plate (of glass) of thickness $d$ and refractive index $n$. Let us call the "main beam" the light that has been refracted at the first surface and again at the second surface. However, some of the light reflected at the second surface may be reflected again at the first surface and then come out at the second one: we will call it the "secondary beam". We postpone the discussion of the higher order beams i.e., those undergoing multiple reflections at both surfaces of the plate. The secondary beam, resulting from two reflections at normal incidence, is retarded relative to the main one with a phase difference

$\phi /(2 \pi)=2 d n / \lambda$

where $\lambda$ is the wavelength in vacuum. We recall that $\cos \phi$ has maxima when $\phi /(2 \pi)=m$ is integer, and minima when $\phi /(2 \pi)=m+1 / 2$. Thus, maxima occur at $\lambda_{m}=2 d n / m$ and 
minima at $\lambda_{m+1 / 2}=2 d n /(m+1 / 2)$. The modulation of the light as a function of $\lambda$ has a period

$P=\lambda_{m}-\lambda_{m+1} \approx \lambda^{2} /(2 d n)$.

According to Fresnel, the reflected beam from a dielectric surface at normal incidence has a reflection coefficient

$R=\frac{\left(n_{1}-n_{2}\right)^{2}}{\left(n_{1}+n_{2}\right)^{2}}$

where $n_{1}, n_{2}$ are the indices of refraction of the two media. For glass/air reflection, with indices $n_{2}=1.5$ and $n_{1}=1$ respectively, one obtains $R=.04$. In the case of a plane parallel glass the relative intensity of the secondary beam is $I_{B} / I_{A} \approx R^{2}$. The third term in Eq. (53), $2 R I_{A} \cos \phi$, indicates now that fringes have quite a significant relative amplitude, $2 R$.

Indeed, the intensity of the fringes is related to the ratio of the amplitudes of the electric vibrations of the two beams $B / A=R=0.04$.

Neglecting the second term in Eq. (53), we can replace this equation by:

$I \approx I_{A}(1+2 R \cos \phi)$.

The demonstration here is only indicative, as several other factors may influence considerably the appearance of fringes. Anti-reflection coating may reduce $R$ by an order of magnitude, the amplitude of the beam $B$ is then reduced by the same factor.

\subsection{Localization and apparent intensities of fringes}

For fringes to be seen in the spectrum, we need to have the main beam $A$ and the secondary beam $B$ in coherence. In our example in the previous subsection we considered a thin and perfect plane parallel plate perpendicular to the light beam. Take now, for instance, a prismatic plate: the fringes are localized on the plate, the direction of propagation of the beam $B$ differs from that of $A$. There is a shift between the images corresponding to two beams when formed on the spectrograph slit, and thus the beams $A$ and $B$ entering the slit are not coherent. Similarly, in the case of solar observations, we cannot bring into coherence beams originating from different parts of the image of the sun. The same occurs with tilted plates: the beam $B$ is displaced relatively to $A$. In thick optical pieces, the additional round trip of the secondary beam causes different results in different focii for $A$ and $B$. Tilted, prismatic or thick plates may reduce or even make disappear fringes. In short, we have not considered the details of the optical set-up: Convergent or divergent beams may exhibit much fainter fringes. A tilt of an optical piece may be effective to remove fringes, but may have other disadvantages. In other words, we may explain the fringes we see, we do not explain the fringes that we do not see, and we have only a relative estimate of the intensity of those fringes. The same comments are relevant also to polarized fringes.

\section{Polarization-dependent fringes}

So far, we sketched the formation of scalar fringes, where the state of polarization was not relevant. We will now turn to the Stokes vector. A Stokes vector must obey the conditions: $I^{2} \geq Q^{2}+U^{2}+V^{2}$, but the ensemble of fringes in all the parameters do not form a four-vector with appropriate conditions for a norm; there may be no Stokes vector representing the polarized fringes. Nevertheless, a Stokes vector describing polarized light may include the effect of fringes in each of its components. Also, an optical device which is at the origin of fringes may be represented by a Mueller matrix operating on Stokes vectors and display the formation of polarized-dependant fringes.

\subsection{On the origins of the polarization in fringes}

Uniaxial crystals are used for both retarders and beam-splitters (analyzers). For the definitions of uniaxial and birefringent crystal, see Hecht (1987) p. 288. These crystals have refraction indices that are a function of the orientation of the vibrations of the electric field relative to the optical axis. For vibrations perpendicular or parallel to the optical axis, the refraction indices are the ordinary $n_{\mathrm{O}}$ and the extraordinary $n_{\mathrm{E}}$, respectively. For an arbitrary direction of vibration the refractive index is intermediate between $n_{\mathrm{O}}$ and $n_{\mathrm{E}}$. Expressions for the indices of refraction of the two vibrations as well as for the phase retardances may be found in Semel (1987). A beam of light passing through a plane parallel plate of a birefringent crystal will be accompanied by secondary beams due to the reflections at the surfaces of the plate. Because of the double refraction, the indices of refraction are dependent on the orientation of the light vibrations and we may anticipate two types of secondary beams - one for each vibration but with different intensity and phase retardation. They yield different fringes in intensity and frequency, and hence polarization-dependent fringes.

\subsection{Analyzers}

There exists a variety of beam-splitters for the purpose of polarization analysis. They often split the light into two orthogonal states, in general linearly polarized and create fringes as well. We will comment here on the simplest and most common.

\subsubsection{The single-piece calcite}

The analyzer, extensively used in the past and described in textbooks on optics consists of a single calcite with its optical axis cut nearly to $45^{\circ}$ with the surface. A beam of light passing through the crystal is split and appears as ordinary and extraordinary rays, in two orthogonal states of linear polarization. The main limitation of this simple solution concerns imaging. Whenever the latter can be neglected (say just one point is analyzed) this device may be safely used. When a two dimensional image has to be analyzed and spatial resolution is important (as in many problems in solar physics) image distortion may be a heavy handicap. Here, we will deal only with the fringes associated with this device. The electric vibrations of the ordinary beam are in the plane perpendicular to the optical axis and those of the extraordinary one are in the plane including this axis. Each of the rays will display fringes with different amplitudes and periods. In calcite, where $n_{\mathrm{O}}>n_{\mathrm{E}}$ the amplitude and the 
period of those pertaining to the ordinary ray are significantly greater than those of the extraordinary one. To calculate the periods of the fringes one can use Eq. (63) and replace $n$ by $n_{\mathrm{O}}$ for the ordinary ray, and by $\hat{n}=n_{\mathrm{O}} n_{\mathrm{E}} / \bar{n}$ for the extraordinary one (see Semel 1987 for the definitions of these terms).

In general the light to be analyzed is processed through a series of unspecified retarders and becomes a Stokes vector $\boldsymbol{I}$ to be analyzed by the beam-splitter into $I_{\mathrm{O}}$ and $I_{\mathrm{E}}$. Neglecting light losses, the ordinary and extraordinary beams may now be replaced respectively by

$I_{\mathrm{O}}\left(1+2 R_{\mathrm{O}} \cos \phi_{\mathrm{O}}\right)$

$I_{\mathrm{E}}\left(1+2 R_{\mathrm{E}} \cos \phi_{\mathrm{E}}\right)$

where $R_{\mathrm{O}}, R_{\mathrm{E}}$ are the reflection coefficients and $\phi_{\mathrm{O}}, \phi_{\mathrm{E}}$ are the retardations for the ordinary and extraordinary beams, while $I_{\mathrm{O}}$ and $I_{\mathrm{E}}$ are the incoming intensities of the ordinary and extraordinary beams respectively.

Polarimetry in this context should be the comparison of the two beams, i.e., $I_{\mathrm{O}}$ and $I_{\mathrm{E}}$, but here, undesired terms appear,namely

$I_{\mathrm{O}} R_{\mathrm{O}} \cos \phi_{\mathrm{O}}$

$I_{\mathrm{E}} R_{\mathrm{E}} \cos \phi_{\mathrm{E}}$

Their sum would be the origin of fringes in the intensity and their difference would be the source of polarized fringes due to this type of beamsplitters.

This single calcite has also several disadvantages as, for instance, different foci for the two rays. It has often been used in the past, but replaced nowadays by better combinations.

\subsubsection{The two crossed crystals (Semel 1980)}

Improved beam-splitters arise from this combination and are currently in use in many astronomical sites. They have better focii for the two beams, that are now both ordinary and extraordinary in a sequential way, as we will see. Moreover, they have a better symmetry since the states of the linear polarization of the two beams are at $45^{\circ}$ with the splitting direction and with the axes of symmetry of the spectrograph, for instance. Residual astigmatism may be reduced by several means, for instance, a special cut creating a small angle of the optical axis with the normal to the surface (see Semel 1987, for other solutions).

Whether these plates are cemented, coated or in immersion, reflections cannot be avoided, since the refractive indices involved are different, corresponding to the ordinary and extraordinary rays of the crystals, the cement used or the immersion liquid. All these create fringes that are polarization-dependent and with quite different frequencies.

We suggest a reference system (not-specified here), where angles may be measured; second, in this system we take the angles: $0^{\circ}$ and $180^{\circ} ; 90^{\circ},-90^{\circ} ; 45^{\circ}$ and $-135^{\circ} ;-45^{\circ}$ and $135^{\circ}$ to correspond to $Q,-Q ; U$ and $-U$,

$\begin{array}{rr}\Phi & \text { Stokes } \\ 0^{\circ} & Q \\ 90^{\circ} & -Q \\ 45^{\circ} & U \\ 135^{\circ} & -U .\end{array}$

A perfect analyzer with a separation of the beams in the direction of $\theta=0^{\circ}, 180^{\circ}$ and the states of polarization at $\pm 45^{\circ}$ (i.e., $\pm U$ ) can be described by the two matrices, for $I \pm U$ :

$A( \pm U)=\frac{1}{2}\left(\begin{array}{cccc}1 & 0 & \pm 1 & 0 \\ 0 & 0 & 0 & 0 \\ \pm 1 & 0 & 1 & 0 \\ 0 & 0 & 0 & 0\end{array}\right)$

For simplicity, we neglect the difference in transmissions of the two beams and concentrate on the formation of polarizationdependent fringes. The ordinary and extraordinary beams $I_{\mathrm{O}}$ and $I_{\mathrm{E}}$ in the preceding example are now replaced by $I_{\mathrm{OE}}$ and $I_{\mathrm{EO}}$. The first, $I_{\mathrm{OE}}$, is the ordinary ray in the first crystal and the extraordinary one in the second one. The second beam $I_{\mathrm{EO}}$ is the converse case. While the two crystals may be similar enough to guarantee imaging and other advantages of this combination, they cannot guarantee the same phases for the various fringes. It is enough that they have a difference of thickness of $0.05 \mu$ for the respective fringes to be shifted relatively by a significant amount.

Similar to the first example we replace the first beam by

$I_{\mathrm{OE}}\left(1+2 R_{\mathrm{O}} \cos \phi_{\mathrm{O} 1}\right)\left(1+2 R_{\mathrm{E}} \cos \phi_{\mathrm{E} 2}\right)$

and the second beam by

$I_{\mathrm{EO}}\left(1+2 R_{\mathrm{E}} \cos \phi_{\mathrm{E} 1}\right)\left(1+2 R_{\mathrm{O}} \cos \phi_{\mathrm{O} 2}\right)$,

with similar meaning as before, except that this time, the indices 1 and 2 point to one or other crystal. $\phi_{\mathrm{O} 2}$ etc. are all different, as explained.

The two Mueller matrices are now:

$$
\begin{gathered}
A(+U)=\frac{1}{2}\left(1+2 R_{\mathrm{O}} \cos \phi_{\mathrm{O} 1}\right)\left(1+2 R_{E} \cos \phi_{\mathrm{E} 2}\right) \\
\times\left(\begin{array}{cccc}
1 & 0 & +1 & 0 \\
0 & 0 & 0 & 0 \\
+1 & 0 & 1 & 0 \\
0 & 0 & 0 & 0
\end{array}\right)
\end{gathered}
$$

and

$$
\begin{gathered}
A(-U)=\frac{1}{2}\left(1+2 R_{\mathrm{E}} \cos \phi_{\mathrm{E} 1}\right)\left(1+2 R_{\mathrm{O}} \cos \phi_{\mathrm{O} 2}\right) \\
\times\left(\begin{array}{cccc}
1 & 0 & -1 & 0 \\
0 & 0 & 0 & 0 \\
-1 & 0 & 1 & 0 \\
0 & 0 & 0 & 0
\end{array}\right) .
\end{gathered}
$$

The two crystalline plates will make possible the appearance of two different systems of polarization-dependent fringes. 


\subsection{Linear retarders and the reconstruction of Mueller matrices}

Assume a pure linear retarder, with the ordinary axis parallel to $x$ and made to increase by $\theta$ the phase difference between the electric vibrations parallel to $x\left(\Phi=0^{\circ}\right), E_{x}$ and those parallel to $y\left(\Phi=90^{\circ}\right), E_{y}$. Neglecting multiple reflection and light losses inside the retarder, the Mueller matrix looks like:

$\Theta=\left(\begin{array}{cccc}1 & 0 & 0 & 0 \\ 0 & 1 & 0 & 0 \\ 0 & 0 & \cos \theta & \sin \theta \\ 0 & 0 & -\sin \theta & \cos \theta\end{array}\right)$.

In the following we will use the abridged notation of $2 \times 2$ matrices,

$\mathcal{M}(\theta)=\left(\begin{array}{cc}\cos \theta & \sin \theta \\ -\sin \theta & \cos \theta\end{array}\right)$,

$\mathcal{N}=\left(\begin{array}{ll}0 & 0 \\ 0 & 0\end{array}\right)$

$\mathcal{U}=\left(\begin{array}{ll}1 & 0 \\ 0 & 1\end{array}\right)$

$\Theta=\left(\begin{array}{cc}\mathcal{U} & \mathcal{N} \\ \mathcal{N} & \mathcal{M}(\theta)\end{array}\right)$

This matrix changes $U$ and $V$ but does not affect $I$ or $Q$ (with the usual notation of the Stokes vector: $(I, Q, U, V)$ ). We now add the effects of double reflections between the two optical surfaces of the retarder and modify the Mueller matrix $\Theta$ to include these effects. First, we calculate the secondary beams for each one of the vibrations. For the $x$ vibrations $\left(E_{x}\right)$ of the secondary beam, the phase difference, according to Eq. (62) is:

$\phi_{\mathrm{O}}=4 \pi d n_{\mathrm{O}} / \lambda$

For the extraordinary vibrations we obtain:

$\phi_{\mathrm{E}}=4 \pi d n_{\mathrm{E}} / \lambda$

The retardance of this plate is simply related to these phases as follows:

$\theta=\left(\phi_{\mathrm{E}}-\phi_{\mathrm{O}}\right) / 2=2 \pi d\left(n_{\mathrm{E}}-n_{\mathrm{O}}\right) / \lambda$.

Note that $\theta$ is the phase retardance of the main beam due to its unique pass through the plate, while $\phi_{\mathrm{E}, \mathrm{O}}$ were defined for a round trip in the plate for the secondary beams, hence the division by 2 in Eq. (82). Let us call

$I_{\text {in }}=(I+Q) / 2$

$I_{y \text { in }}=(I-Q) / 2$

the incoming intensities of the vibrations parallel to $x$ and $y$ which may also be called ordinary and extraordinary, respectively. Also we call $R_{\mathrm{O}}$ and $R_{\mathrm{E}}$ the coefficients of reflection at the plate surface for the two beams $\mathrm{O}$ and $\mathrm{E}$, respectively. They may be different since the indices that enter the Fresnel formula are not the same for the ordinary and extraordinary beams.
Including the effects to the first order in $R_{\mathrm{O}}$ and $R_{\mathrm{E}}$, the outgoing intensities are (light losses at the plate surfaces included):

$I_{x \text { out }}=I_{x \text { in }}\left[1+2 R_{\mathrm{O}}\left(-1+\cos \phi_{\mathrm{O}}\right)\right]$

for the $x$ or $\mathrm{O}$ vibrations and

$I_{y \text { out }}=I_{y \text { in }}\left[1+2 R_{\mathrm{E}}\left(-1+\cos \phi_{\mathrm{E}}\right)\right]$

for the $y$ or E vibrations.

Let us call the outgoing Stokes parameters $I_{\text {out }}, Q_{\text {out }}$ etc. Then

$I_{\text {out }}=I_{x o u t}+I_{y \text { out }}$

$Q_{\text {out }}=I_{\text {xout }}-I_{\text {yout }}$.

We have to modify the matrix $\Theta$ in Eq. (75) to include these effects as follows:

$\Theta_{11}=\Theta_{22}=\left(1+R_{\mathrm{O}}\left(\cos \phi_{\mathrm{O}}-1\right)+R_{\mathrm{E}}\left(\cos \phi_{\mathrm{E}}-1\right)\right)$

$\left.\Theta_{12}=\Theta_{21}=R_{\mathrm{O}}\left(\cos \phi_{\mathrm{O}}-1\right)-R_{\mathrm{E}}\left(\cos \phi_{\mathrm{E}}-1\right)\right)$.

When $\left(n_{\mathrm{E}}-n_{\mathrm{O}}\right)$ is small we may anticipate that

$R_{\mathrm{O}} \approx R_{\mathrm{E}}$, and therefore

$\Theta_{11}=\Theta_{22} \approx 1-2 R_{\mathrm{O}}+R_{\mathrm{O}}\left(\cos \phi_{\mathrm{E}}+\cos \phi_{\mathrm{O}}\right)$

$\Theta_{12}=\Theta_{21} \approx R_{\mathrm{O}}\left(\cos \phi_{\mathrm{E}}-\cos \phi_{\mathrm{O}}\right)$.

For a quarter wave plate one has $\theta=\pi / 2$ and therefore, in virtue of Eq. (82), $\cos \phi_{\mathrm{O}}=-\cos \phi_{\mathrm{E}}$. Thus $\Theta_{11}(\pi / 2)=$ $\Theta_{22}(\pi / 2)$ are free of fringes but $\Theta_{12}(\pi / 2)=\Theta_{21}(\pi / 2)$ are rich sources of fringes, mainly in $Q$ "contributed" by $I$.

On the contrary, in a half wave plate, $\theta=\pi$, the fringes appear in the intensity $I$ only, as well as in the case of a full wave plate. The other Stokes parameters are free of fringes. If a polarimeter includes several retarders, one may expect all kinds of fringes, in any of the Stokes parameters and (or) in linear combinations of them. These fringes do depend on the orientations of the retarders and change upon rotation. (This has a serious bearing on the actual polarimetry.)

The other terms in the matrix $\Theta$ are also affected by the double reflection. To show this, one has to consider the new modified phases and amplitudes upon the addition of the secondary beam.

We apply Eqs. (58) and (61) to our retarder, and, to the first order in $R_{\mathrm{O}}$ and $R_{\mathrm{E}}$, the amplitudes of the ordinary and extraordinary vibrations are modified by the factors:

$\left[1+R_{\mathrm{O}}\left(\cos \phi_{\mathrm{O}}-1\right)\right] \quad$ and $\quad\left[1+R_{\mathrm{E}}\left(\cos \phi_{\mathrm{E}}-1\right)\right]$

respectively.

The terms $\cos \theta$ and $\sin \theta$ in the matrix $\Theta$ should be therefore multiplied by

$\left[1+R_{\mathrm{O}}\left(\cos \phi_{\mathrm{O}}-1\right)\right]\left[1+R_{\mathrm{E}}\left(\cos \phi_{\mathrm{E}}-1\right)\right]$

the angle $\theta$ should be replaced by

$\theta+R_{\mathrm{E}} \sin \phi_{\mathrm{E}}-R_{\mathrm{O}} \sin \phi_{\mathrm{O}}$. 
This represents a quite different kind of periodicity, in a new dimension: fluctuations of the retardance with wavelength, see Holmes (1964). Indeed, it is known as the effect of multiple reflection on the retardation.

For illustration, we present here the calculation of these effects for a particular orientation of the device. Note that the matrix constructed is still a Mueller one and is subject to rotation through the usual matrix algebra. (See Shurcliff 1966, p. 116.)

\section{The achromatic quarter wave plate - the Fichou type in the visible}

In the case of a perfect $\lambda / 4$ plate, one has

$$
\Theta=\left(\begin{array}{cccc}
1 & 0 & 0 & 0 \\
0 & 1 & 0 & 0 \\
0 & 0 & 0 & 1 \\
0 & 0 & -1 & 0
\end{array}\right) .
$$

However, in practice we can only approach it. Fichou's commercial achromatic $\lambda / 4$ is commonly used in astrophysics and deserves special attention ${ }^{1}$.

It is composed of two plane parallel uniaxial crystals $\mathrm{MgF}_{2}$ and Quartz. They are made to compensate the wavelength dependence and obtain a nearly achromatic plate in the visible. Let us represent the incoming light by the components of its electric vector along the $x$ and $y$ axes, as

$E_{x} \cos (\omega t)$

$E_{y} \cos (\omega t+\psi)$

where $\omega$ is the angular frequency and $\psi$ the phase difference between the two components. After passing through a uniaxial crystal plate the components are:

$$
\begin{aligned}
& E_{x}\left[\cos \left(\omega t-\phi_{x}\right)+\alpha_{x}\left(\cos \left(\omega t-3 \phi_{x}\right)\right]\right. \\
& E_{y}\left[\cos \left(\omega t+\psi-\phi_{y}\right)+\alpha_{y} \cos \left(\omega t+\psi-3 \phi_{y}\right)\right]
\end{aligned}
$$

where the retardation phase changes are, respectively

$$
\begin{aligned}
& \phi_{x}=2 \pi n_{x} d / \lambda \\
& \phi_{y}=2 \pi n_{y} d / \lambda
\end{aligned}
$$

$n_{x, y}$ being the respective indices of refractions and $d$ the thickness of the plate. We neglect the losses of the main beams but consider the contribution of the additional beams, due to reflections at each surface, with the relative amplitudes $\alpha_{x, y}<10^{-2}$, (the relative smallness is due to anti-reflection coatings, usually applied to these commercial achromatic retarders). Since these secondary beams pass the plate three times, the phase retardation is, consequently, three times that of the main beam. Higher order beams are neglected. The two electric components are now approximated by:

$$
\begin{aligned}
& E_{x}\left(1+\alpha_{x} \cos 2 \phi_{x}\right) \cos \left(\omega t-\phi_{x}+\phi_{x x}\right), \\
& E_{y}\left(1+\alpha_{y} \cos 2 \phi_{y}\right) \cos \left(\omega t+\psi-\phi_{y}+\phi_{y y}\right),
\end{aligned}
$$

\footnotetext{
${ }^{1}$ Optique de Précision. Jean Fichou, 30 rue de la Garenne, 94260 Fresnes, France.
}

where

$\sin \phi_{x x}=-\alpha_{x} \sin 2 \phi_{x}$.

A similar expression holds for $\phi_{y y}$. The effects of these terms will be additional small fluctuations of the retardation phase. We now construct the Mueller matrix that describes the effects of this uniaxial plate. From the last expressions, we derive the factors by which the amplitudes of the electric vibrations change: for the $x$ and $y$ vibrations:

$C_{x}=\left(1+\alpha_{x} \cos 2 \phi_{x}\right)$

$C_{y}=\left(1+\alpha_{y} \cos 2 \phi_{y}\right)$.

At the same time, the phase difference between the two vibrations changes by the amount of:

$\gamma=-\phi_{y}+\phi_{y y}-\left(-\phi_{x}+\phi_{x x}\right)$.

In order to replace $\mathcal{U}$ in Eq. (78), it is convenient to define a sub-matrix $C_{X, Y}$ as follows:

$C_{X, Y}=\frac{1}{2}\left(\begin{array}{ll}C_{x}^{2}+C_{y}^{2} & C_{x}^{2}-C_{y}^{2} \\ C_{x}^{2}-C_{y}^{2} & C_{x}^{2}+C_{y}^{2}\end{array}\right)$,

Then, one obtains the compact presentation to replace Eq. (79):

$\Theta=\left(\begin{array}{cc}\mathcal{C}_{X, Y} & \mathcal{N} \\ \mathcal{N} & C_{x} C_{y} \mathcal{M}(-\gamma)\end{array}\right)$.

One can easily verify that in absence of reflections, i.e., $\alpha_{x}=$ $\alpha_{y}=0$ the last matrix is reduced to the standard Mueller matrix of a linear retarder. However, in the case of very low polarization the cross-talk from $I$ to $Q$ in the shape of fringes may become very important. To complete the construction of the achromatic $\lambda / 4$ one has to "add" the two plates. Thus, we denote a Mueller matrix $\Theta_{M}$ for the crystal $\mathrm{MgF}_{2}$ and $\Theta_{\mathrm{Q}}$ for the quartz. According to the instructions of the maker we should allow for a possible misalignment of the order of $15 \mathrm{~min}$. The thickness of the two plates $\left(d_{\mathrm{M}}=187.9 \mu\right.$ and $\left.d_{\mathrm{Q}}=227.1 \mu\right)$ is chosen so as to obtain by subtraction $90^{\circ}$ retardation at $\lambda=425$ and $625 \mathrm{~nm}$. In between, the deviation may reach $4.5^{\circ}$ at most.

Here, we will evaluate each of the effects as if it were possible to neglect all the others. This is justified because all of the effects are small. First, we will present the result of the misalignment. Next, we will assume perfect alignment and consider the two plates as if they were cemented and that the phase differences are the results of the algebraic addition of the phases of the individual plates and thus given by:

$\phi_{x}=\phi_{x \mathrm{M}}+\phi_{x \mathrm{Q}}$

$\phi_{y}=\phi_{y \mathrm{M}}+\phi_{y \mathrm{Q}}$

where the indices $\mathrm{M}$ and $\mathrm{Q}$ stand for $\mathrm{MgF}_{2}$ and Quartz, respectively. The two plates being crossed, the ordinary ray for the first plate is the extraordinary for the second. The resultant $\gamma$ corresponds approximately to a quarter wave plate,

$\gamma \approx \pi / 2$

and in the following we will assume that

$\phi_{x}-\phi_{y} \approx \pi / 2$. 
With the approximation

$\alpha_{x} \approx \alpha_{y}=\alpha$

we obtain

$C_{x}^{2}+C_{y}^{2} \approx 2$

$C_{x}^{2}-C_{y}^{2} \approx-2 \alpha \sin \left(\phi_{x}+\phi_{y}\right)$.

We now define

$\beta=-\alpha \sin \left(\phi_{x}+\phi_{y}\right)$,

and use the relation

$C_{x} C_{y} \approx 1$.

We abridge

$\mathcal{U}_{\beta}=\left(\begin{array}{cc}1 & \beta \\ \beta & 1\end{array}\right)$

to obtain finally,

$\Theta=\left(\begin{array}{cc}\mathcal{U}_{\beta} & \mathcal{N} \\ \mathcal{N} & \mathcal{M}(-\gamma)\end{array}\right)$

The term that causes linearly polarized fringes parallel to the optical axis is $\beta$. At $\lambda=5000 \AA$, the expected periods are roughly $3.5 \AA$ for the Quartz and $4.8 \AA$ for $\mathrm{MgF}_{2}$. The differences of periods of the ordinary and extraordinary rays are roughly $\Delta P_{\mathrm{Q}}=0.02 \AA$ for Quartz and $\Delta P_{\mathrm{M}}=0.02 \AA$ for $\mathrm{MgF}_{2}$. These differences correspond to fringes created in each of the crystals separately. When the crystals are cemented, the fringes created by the outer surfaces have roughly the period of $2 \AA$.

We can comment here on the different effects present (see Appendix B):

- Alignment: The axis of the composite $\lambda / 4$ is $\lambda$ dependent and it will pivot around the axis of the second plate encountered by the light in a swing of $\pm 15 \mathrm{~min}$, at least.

- Internal reflections: It may be the most troublesome, mainly in the case of weak polarization. The fringes in linear polarization parallel (or perpendicular) to the axis of the crystals, with a relative amplitude $\alpha$ of the order of $10^{-3}$ to $10^{-2}$, may be hard to remove. The usual technique of flat-fielding may be of great help, but will fail if the flat-field exposures are distant in time from the observation. If, for instance, each plate is cut and polished to $\lambda / 10$, the fluctuations of the thickness $d$ are

$\delta d \approx \pm \lambda /(10(n-1))$

and the corresponding phase differences, in units of $\lambda$, are

$\delta \phi=2 \delta d n / \lambda= \pm 2 n /(10(n-1))$.

For $n=1.5, \delta \phi \approx \pm 0.6$ there are little or no phase correlations for fringes due to different parts of the $\lambda / 4$ plate. The frequency of the fringes might be the same all over the $\lambda / 4$ retarder, but the phase may fluctuate by \pm 200 degrees. In other words the fringes are the same for two exposures only if the light goes through the same part of the plate each time.

- Fluctuations in retardation they are due to multiple reflections, Holmes (1964).

\section{Conclusions}

1. For fringes to appear, we need two coherent beams that may interfere. We anticipater that optical path differences between the two beams lead to positive and negative interferences successively and create fringes.

2. In this paper we first discussed the formation of fringes in a two beam set-up as introduced by Young and realized by Fresnel, Arago and many others. In this first type of fringes, the path differences (and phases) are due to geometrical differences.

3. When the beams are coherent, the two perpendicular vibrations of the light may or may not have a phase relation (the condition necessary for fringes). When the two beams are linearly polarized in orthogonal states, there will be no fringes in intensity, in full agreement with the FresnelArago law. But polarized fringes are possible, depending on the polarization of the light at the source.

4. Finally, we simulate a new "experiment" that it shows fringes of an intermediate character between the FresnelArago type and the multiple reflection type which is the subject of this paper.

5. The second type of interference, and the resulting fringes, are of a quite different nature. Typically, multiple reflections in a plane parallel plate create secondary beams with phase differences due the excess of crossings inside the plate. Scalar fringes are described first. Next, we find a transition to polarized fringes.

6. In birefringent media and, in general in all retarders a quite different process is invoked. The eigenstates of the light in general are polarized in anisotropic media. They also have different light velocities. Each eigenstate develops its own pattern of fringes. The sum of the two eigenstates may show fringes in intensity or in polarization depending on the frequencies and the phases of the fringe pattern of each one of the eigenstates. Particularly, their phase fluctuates considerably over each of the optical components. Therefore, the necessary condition for using flat-fielding techniques is that the light beams during the exposures for flat-fielding and for real observations go in an identical way through all the optical components. It is therefore desired to develop an adequate concept of polarimetry for both solar and stellar objects; the calculations presented here will prove to be useful for this purpose. One may anticipate the successful use of the concept of polarimeters free of fringes and the establishment of suitable observational procedures for the removal of fringes in actual polarimeters.

\section{Epilogue: Stokes polarimetry, fringes and the related phase relations}

Stokes polarimetry requires the consideration of three categories of phase relations:

1. The historical definition of a Stokes vector: phase relations are defined locally and simultaneously between orthogonal components of the light and lead to the usual Stokes vector (or parameters). The polarization in the stellar 
atmosphere is due to various physical process like light diffusion, Zeeman and Hanle effects and impact polarization.

2. Phase modifications are introduced successively or nonlocally to perform measurements. The technology of polarimetry does not simulate the phase relations as in the first kind, but it changes the state of polarization by using Mueller matrices to allow for the desired measurements.

3. Interference effects (and polarized fringes) due to unavoidable secondary beams in the optics depend on the phase relations. Here, the phase relations concern any component in one of the beams with any other component in another beam, even in the absence of any particular polarization. Usually the two interfering beams are originally beamsplit from one original. The phase differences may be due to optical effects in the set up.

It looks like most of the efforts and attention of the polarimetrists are directed to the formalism of Stokes vectors and Mueller matrices: the formalism of the Minkowski space López (1999). Indeed, it is a fancy space in Wonderland governed by elegant and sophisticated algebra. Prisoner of this formalism, the polarimetrist neglects the other optical effects. We find no comments on polarization dependent fringes in books like Shurcliff (1966). True, the problem does not appear in broad band polarimetry. However, nature speaks also in the language of polarized fringes and we must admit that the realm of light interferences and phase relations concerns us as well when we use spectropolarimetry in high spectral resolutions. It may be claimed that the Jones calculus takes into account any interference effect. As will be shown in Appendix C, the Jones calculus is definitely insufficient and does not solve the problem.

Now, when we turn to the spectropolarimetric measurements we must face considerations that do not appear in either Stokes or Jones calculus.

When we contemplate spectropolarimetric measurements, we must admit two different methods: 1) the common destructive methods, 2) the non-destructive methods. Minkowski space, where four-vectors are best described, does not postulate how to measure Stokes Vectors.

1) The common destructive methods By using a linear polarizer we may measure, for instance, $I+Q$. By using a beamsplitter we may even measure simultaneously two beams $I \pm Q$. After the measurements we lose the light. In order to measure all Stokes parameters, we need to repeat the measurements for $I \pm U$ and $I \pm V$, assuming that the source is constant, including atmospheric and instrumentation effects. We call these destructive methods because after each operation the light is lost. Can we measure each of the $Q, U$ and $V$ directly inside the Minkowski space and keep the light?

2) The non-destructive option

In principle it is possible. When a beam of light goes through a $\lambda / 2$ plate, its $V$ and therefore also its angular momentum changes its sign. By the physical principle of angular momentum conservation, the $\lambda / 2$ plate has acquired the angular momentum corresponding to $2 \mathrm{~V}$. We may measure it and not lose the light. Therefore, we call the measurement non-destructive. The physics is perfect, but there is no industry to invent the instrumentation. We are not able to assess the methods without intensive experimentation.
But, even the destructive methods have their shortcomings, constraints and parasite effects like polarized fringes.

Acknowledgements. The author thanks Prof. Sami Cuperman for reading the first version, is indebted to Véronique Bommier for the full verification of all equations. He thanks Jean-Louis Leroy for encouragement and clarifying important concepts and acknowledges the contribution of Pierre Bastien that led to an important extension of the last version. He is thankful particularly to Arturo López Ariste who carefully corrected the last manuscript and to Nisan Spector for a last reading.

\section{Appendix A: A justification of the application of the scalar method to polarized fringes}

For calculations of phases, one may use each one of the eigenstates as if it were propagating in an isotropic plate, but with the adequate refraction coefficient or light velocity. The term eigenstate of light needs to be explained. Let us take, for instance, a quarter wave plate: a beam of light may penetrate the plate in a circular polarization state and exit in a linear one. Circular polarization is, therefore, not an eigenstate in this case. When the linear polarization is parallel, either to the fast or to the slow axis, it travels unchanged, while for any other orientation it will come out elliptic. Thus a linear retarder has two eigenstates of linear polarization, one parallel and another one perpendicular to the optical axis. The ordinary beam (electric vector always perpendicular to the optical axis of the crystal) propagates according to the same rules as for light in isotropic media and Sect. 3 applies.

The extraordinary beam is still linearly polarized. However, the refraction is not the same as for isotropic media, also the velocity of light is direction-dependent. It is easy to find out experimentally what the eigenstates in question are. The optical piece in question is placed between two linear polarizers that are turned until extinction is obtained. Its level can reach $10^{-5}$ with little difficulty depending on the quality of the polarizers and the alignment. In the limits of normal incidence on retarders, the method is perfect.

\section{Appendix B: Some properties of the Fichou's achromatic $\lambda / 4$ plate}

We skip the case of cemented plate and discuss the laser quality $\lambda / 4$ case. Following the discussion in Sect. 5 , we may integrate all the defects. Thus the total matrix $\Theta_{\mathrm{T}}$ should look like:

$\Theta_{\mathrm{T}}=\left[\Phi_{\mathrm{M}}\right]\left[\Theta_{\mathrm{M}}\right]\left[\Phi_{\mathrm{M}}\right]^{-1}\left[\Phi_{\mathrm{Q}}\right]\left[\Theta_{\mathrm{Q}}\right]\left[\Phi_{\mathrm{Q}}\right]^{-1}$.

Assuming that all errors are small, we keep only linear terms and neglect quadratic or mixed terms. The last expression may be developed easily as follows: If the alignment were perfect, the matrices $\left[\Theta_{\mathrm{M}}\right]$ and $\left[\Theta_{\mathrm{Q}}\right]$ should be in orthogonal orientations for subtraction, i.e. at $90^{\circ}$. However a mis-alignment of $2 \delta$ is considered with the following symmetric orientations: the two crystals are misaligned relatively to the zero-axis (or axis of symmetry) by $\pm \delta$ :

$\left[\Phi_{\mathrm{M}}\right]^{-1}=\left[\Phi_{\mathrm{Q}}\right]=[\delta] \approx\left(\begin{array}{cccc}1 & 0 & 0 & 0 \\ 0 & 1 & 2 \delta & 0 \\ 0 & -2 \delta & 1 & 0 \\ 0 & 0 & 0 & 1\end{array}\right)$ 


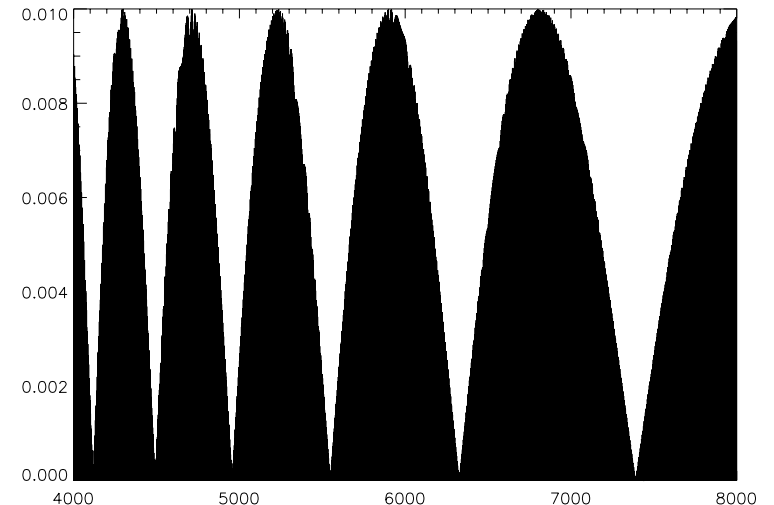

Fig. B.1. The absolute value of the fringes in Stokes I, between 4000 and $8000 \AA$ as created by the quartz plate. Having too small periods ( 3 to $6 \AA$ roughly) to be resolved in the figure, the bulk of fringes coincide with the dark area. The amplitude, at the peak, was taken arbitrarily to $1 \%$ of the local intensity (i.e. of the Stokes parameter $I$ ), and for both $I$ and $Q_{0}$ in all figures.

where for $|\delta| \ll 1$, we have substituted $\cos \delta \approx 1$ and $\sin \delta \approx \delta$. Thus Eq. (B.1) reads:

$\Theta_{\mathrm{T}}=[-\delta]\left[\Theta_{\mathrm{M}}\right][2 \delta]\left[\Theta_{\mathrm{Q}}\right][-\delta]$.

The effect of $\delta$ has been explained in the first item in Sect. 5 as the pivoting of the axis of the $\lambda / 4$ plate with wavelength.

We therefore continue with the other effects by neglecting $\delta$ and beyond the pivot effect:

$\Theta_{\mathrm{T}} \approx\left[\Theta_{\mathrm{M}}\right]\left[\Theta_{\mathrm{Q}}\right]$.

If we neglect fringes for a moment, and we assume that $\Theta_{T}$ should be a nearly perfect $\lambda / 4$ plate, with a small error of retardance $\varepsilon$ :

$\Theta_{\mathrm{T}} \approx\left(\begin{array}{cccc}1 & 0 & 0 & 0 \\ 0 & 1 & 0 & 0 \\ 0 & 0 & \cos (\pi / 2+\varepsilon) & \sin (\pi / 2+\varepsilon) \\ 0 & 0 & -\sin (\pi / 2+\varepsilon) & \cos (\pi / 2+\varepsilon)\end{array}\right)$

that we approximate

$\Theta_{\mathrm{T}} \approx\left(\begin{array}{cccc}1 & 0 & 0 & 0 \\ 0 & 1 & 0 & 0 \\ 0 & 0 & 0 & 1 \\ 0 & 0 & -1 & 0\end{array}\right)-\left(\begin{array}{llll}0 & 0 & 0 & 0 \\ 0 & 0 & 0 & 0 \\ 0 & 0 & \varepsilon & 0 \\ 0 & 0 & 0 & \varepsilon\end{array}\right)$.

The first matrix is the ideal $\lambda / 4$ plate, let us call it the ideal or the [SOFT] matrix, the one that is mentioned in textbooks. The second matrix, $[\varepsilon]$, describes the effect of a small excess of retardation. The effects due to multiple reflections, i.e. fringes and the modification of the retardation due to each of the crystals, may be expressed by the difference of the matrix $\Theta$ in Eq. (108) and the matrix [SOFT]. Here, we are interested in particular in fringes. They are best expressed by the difference sub-matrix for quartz when the entries in $C_{X, Y}$, Eq. (107), are taken for this crystal: $[\mathrm{SUB}]_{\mathrm{Q}}=C_{X, Y}-\mathcal{U}$, and similarly $[\mathrm{SUB}]_{\mathrm{M}}$ for the other one. We then define the total fringes matrix for quartz as

$\mathcal{F}_{Q}=\left(\begin{array}{cc}{[\mathrm{SUB}]_{\mathrm{Q}}} & \mathcal{U} \\ \mathcal{U} & \mathcal{U}\end{array}\right)$

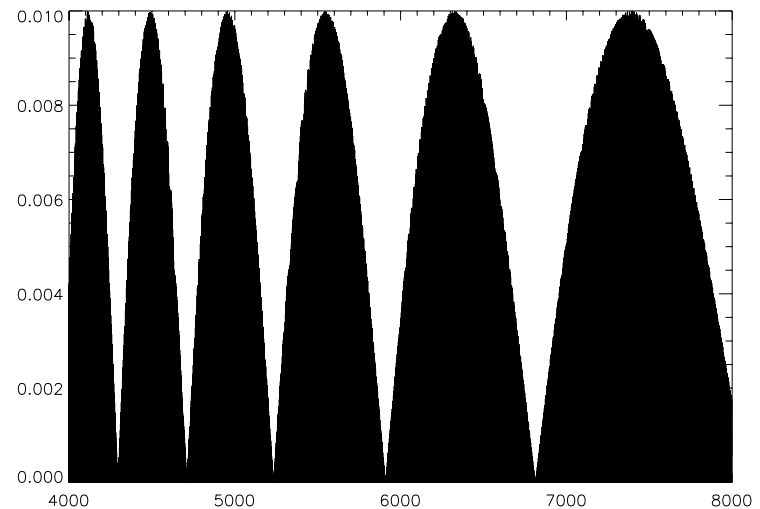

Fig. B.2. The same as Fig. B.1 but for Stokes $Q_{0}$. Note the shift of the envelopes for fringes in Stokes $I$ and $Q_{0}$.

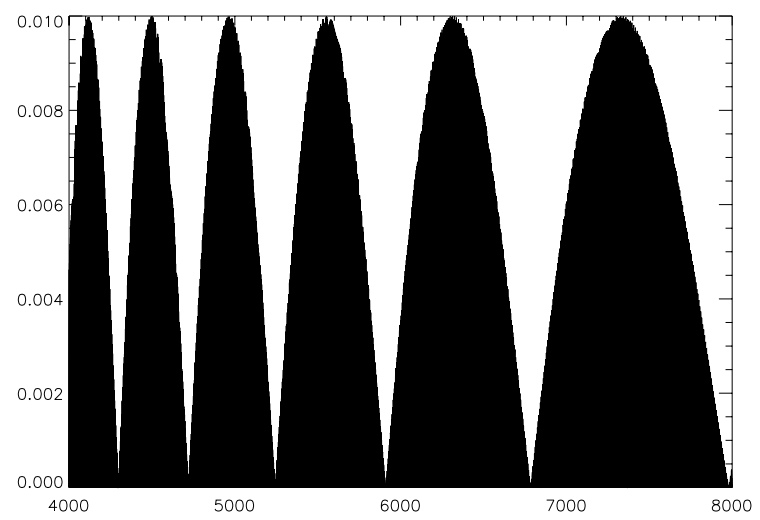

Fig. B.3. The same as Fig. B.1 for the Stokes I, but for the $\mathrm{MgF}_{2}$ plate.

and the same for $\mathcal{F}_{\mathcal{M}}$. The total fringes for Fichou's $\lambda / 4$ plate are $\mathcal{F}_{\mathcal{F}}=\mathcal{F}_{Q}-\mathcal{F}_{\mathcal{M}}$. The difference stands for the subtraction of the orientations of the two crystals. The sign of $\mathcal{F}_{\mathcal{F}}$ is immaterial since the phase of the fringes is not relevant. To the level of accuracy needed here we may approximate Eq. (B.1) by

$\Theta_{\mathrm{T}}=[\mathrm{SOFT}]-[\varepsilon]+\mathcal{F}_{\mathcal{F}}+\delta$ effects.

\section{B.1. More on $\delta$ effects}

We remove all the other effects and keep only those due $\delta$. Originally the rotation matrix read:

$\left[R_{\delta}\right]=\left(\begin{array}{cccc}1 & 0 & 0 & 0 \\ 0 & \cos 2 \delta & \sin 2 \delta & 0 \\ 0 & -\sin 2 \delta & \cos 2 \delta & 0 \\ 0 & 0 & 0 & 1\end{array}\right)$.

We keep the approximations $\cos (2 \delta) \approx 1$ and $\sin (2 \delta) \approx 2 \delta$ Also, we define $[\delta]_{0}=\left[R_{\delta}\right]-[1]$ and we get

$[\delta]_{0}=2 \delta\left(\begin{array}{cccc}0 & 0 & 0 & 0 \\ 0 & 0 & 1 & 0 \\ 0 & -1 & 0 & 0 \\ 0 & 0 & 0 & 0\end{array}\right)$ 


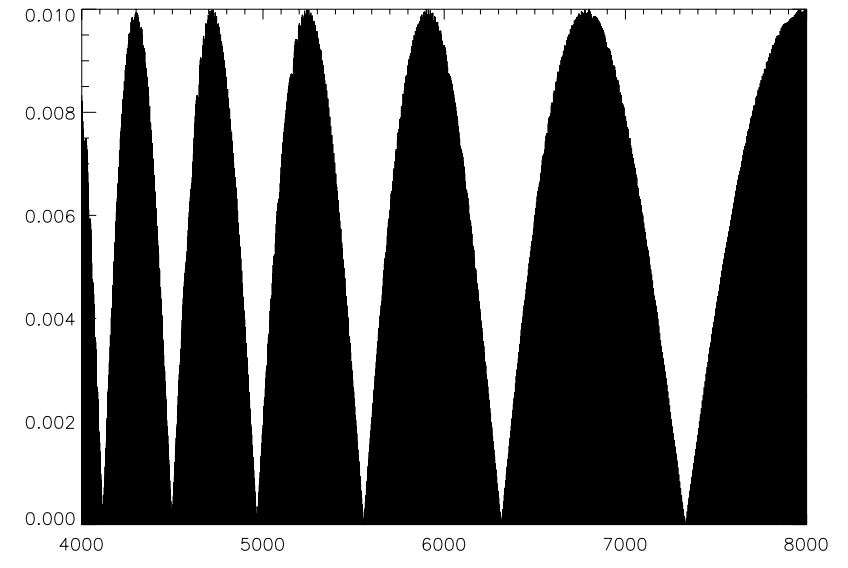

Fig. B.4. The same as Fig. B.1 for Stokes $Q_{0}$, due to the $\mathrm{MgF}_{2}$ plate. Note the similarities of the Figs. B.1 and B.4 on one hand and of B.2 and B. 3 on the other hand.

We remove all the other effects and keep only those due to $\delta$ :

$[\delta]_{0}[\mathrm{SOFT}]+[\mathrm{SOFT}][\delta]_{0}-2\left[\Phi_{\mathrm{M}}\right][\delta]_{0}\left[\Phi_{\mathrm{Q}}\right]$

$=2 \delta\left(\begin{array}{cccc}0 & 0 & 0 & 0 \\ 0 & 0 & -S_{\mathrm{M}} & -C_{\mathrm{M}} \\ 0 & C_{\mathrm{M}} & 0 & 0 \\ 0 & -S_{\mathrm{M}} & 0 & 0\end{array}\right)$

where

$S_{\mathrm{M}}=2 \sin \phi_{\mathrm{M}}-1$

$C_{\mathrm{M}}=2 \cos \phi_{\mathrm{M}}-1$

and $\phi_{\mathrm{M}}$ is the retardation of the $\mathrm{MgF}_{2}$ plate.

In Figs. B. 1 and B. 2 we plot the fringes in $I$ and $Q_{0}$ arising from a Fichou's $\lambda / 4$ achromatic visible and laser type (noncemented plates). We take the interesting case where the incident beam is completely unpolarized. Fringes appear in both $I$ and $Q_{0}$, but there are no fringes in $U_{0}$ or $V$. Here we use the subindex $Q_{0}$ and $U_{0}$ to remind that they refer to the axis of the $\lambda / 4$ plate: $\pm Q_{0}$ are parallel and perpendicular to this axis. But these axes may coincide with those of $Q$ and $U$ in our polarimeter. Therefore, $U_{0}$ has no fringes, while $U$ may have them as a result the rotation between the two different reference systems, etc.

\section{References}

Born, M., \& Wolf, E. 1999, Principle of Optics, 7th edn. (Cambridge University Press)

Donati, J-F., \& Wade, G. A. 1999, A\&A, 341, 216

Fresnel, A. J. 1816, Ann. Chim. (Paris) [2] 1, 239

Hecht, E. 1987, Optics (Addison-Wesley)

Holmes, J. 1964, Opt. Soc. Amer., 54, 1115

Jenkins, F. A., \& White, H. E. 1957, Fundamentals of Optics (McGraw-Hill)

Leroy, J-L. 1998, La polarization de la lumière et l'observation astronomique (Gordon and Breach Science Publishers)

Lites, B. W. 1991, in Solar Polarimetry, Proc. of 11th NSO/Sac Peak summer workshop, 166

Leroy, J-L. 2002, private communication

López Ariste, A. 1999, Thesis, Université Paris 7

López Ariste, A. 2002, private communication

Makita, M., Hamana, S., Kawakami, H., \& Nishi, K. 1982, Ann. Tokyo Ast. Obs., 19, 24

Mein, P., \& Rayrole, J. 1985, Vistas Astron., 28, 567

Semel, M. 1980, A\&A, 91, 369

Semel, M. 1987, A\&A, 178, 257

Shurcliff, W. A. 1966, Polarized light (Cambridge, Massachusetts: Harvard University Press) 181

Skumanich, A., \& Lites, B. W. 1987, ApJ, 322, 473

del Toro Iniesta, J. C., \& Collados, M. 2000, Appl. Opt., 39, 1637

Rees, D. E., Murphy, G. A., \& Durrant, C. J. 1989, ApJ, 339,1093

Unno, W. 1956, PASJ, 1956, 8, 108

Wood, R. W., 1911, Physical Optics (The Macmillan company), 151

Wood, R. W., 1904, April, Phil. Mag. 